MATEO, G. y M.B. CRESPO -1990-Claves para la Flora Valenciana. Ed. Del Cenia al Segura. MONTENEGRO, G., S. TEILLIER, P. ARCE \& V. POBLETE - 1991- Introduction of plants into the mediterranean-type climate area of Chile. En Groves, R.H. \& F. Di Castri (eds.), Biogeography of Mediterranean Invasions. Cambridge Univesity Press.

QUÉZEL, P. \& S. SANTA -1962- Nouvelle Flore de l'Algérie et des régions désertiques méridionales, vol. I. Ed. Centre National de la Recherche Scientifique. París.

RATTER, J.A. -1990- Spergula L. En Castroviejo, S., Laínz, M., López-González, G., Monserrat, P., Muñoz-Garmendia, F., Paiva, J. \& Villar, L. (eds.), Flora Ibérica, vol. II. Real Jardín Botánico de Madrid. C.S.I.C.

SMITH, J. \& T.H. TUTIN - 1968-Euphorbia L. En Tutin, T.H., Heywood, V., Burguess, N., Moore, D., Valentine, D., Walters, S. \& Webb, D. (eds.) Flora Europaea, vol. 2.
TRABUT, L. - 1904- Naturalisation de deux Atriplex australiens dans le Nord de'l Afrique $(A$. halimoides Lindl., A. semibaccata R.Br.). Bull. Société Bot. France 51: 105-106.

VALDÉS, B. -1987- Euphorbia L. En VALDÉs, B., Talavera, S. \& Galiano, E. (eds.)Flora Vasular de Andalucía Occidental, vol. 2.

Aceptado para su publicación en Junio de 1998

Dirección de los autores. Dpto. de Biología Vegetal y Ecología. Universidad de Almería. E04120Almería. España.

\title{
60. APORTACIONES A LA FLORA VASCULAR DE SIERRA NEVADA (ALMERÍA): EL ELEMENTO MURCIANO-ALMERIENSE
}

\author{
Juan LORITE MORENO, Francisco Bruno NAVARRO REYES \\ y Francisco VALLE TENDERO
}

New records for the vascular flora of Sierra Nevada (Almería): The Murciano-almeriense element.

Palabras clave. Flora, Sierra Nevada, Almería, Murciano-almeriense.

Key Words. Flora, Sierra Nevada, Almería, Murciano-almeriense.

Como resultado de los trabajos que estamos realizando sobre flora y vegetación de la Sierra Nevada Almeriense, se aportan citas de trece especies nuevas para el catálogo florístico de Sierra Nevada. Estas citas suponen un importante contingente murcianoalmeriense que caracteriza florísticamente la zona suroriental del parque. Si tenemos en cuenta que muchos de ellos son endemismos de área bastante restringida, o cuando menos 
elementos raros, su presencia tiene importantes connotaciones de cara a la gestión de este espacio natural.

La nomenclatura y autorías de los táxones corresponden con las recogidas en Flora Ibérica (Castroviejo et al., 1986-1997) y Flora Europaea (Tutin et al., 1964-1980), para la corología se han consultado fundamentalmente los trabajos de: Molero et Pérez-Raya, 1987; Mota et Valle, 1987; Sagredo, 1987; MoleroMesa et al., 1992).

Los pliegos testigo se encuentran depositados en herbario de la Facultad de Ciencias de la Universidad de Granada (GDAC).

Para caracterizar las comunidades en las que se hallan incluidas las especies, nos hemos basado en numerosos inventarios realizados en la zona. Tenemos que puntualizar que para las especies que no son endémicas de la provincia murciano-almeriense, la ecología se refiere a la observada en las comunidades de la zona de estudio o zonas adyacentes. En cuanto a la fitogeografía, las unidades de referencia son las recogidas por Rivas-Martínez et al. (1997).

\section{Anthyllis terniflora (Lag) Pau}

ALMERÍA: Rambla del Arquillo, Alboloduy. 30S WF3098, $550 \mathrm{~m}$, en tomillares semiáridos, I. Bocio, J. Lorite et F. Valle, 20-4-95, GDAC 42361. Rambla de los Yesos, Alboloduy. 30SWF3199, 600 $\mathrm{m}$, en tomillares semiáridos, I. Bocio, J. Lorite et $\mathrm{F}$. Valle, 20-4-95, GDAC 42362. Prox. de Alsodux. 30S WF3098, $350 \mathrm{~m}$, en tomillares semiáridos, J. Lorite, 27-4-96, GDAC 42363.

Endemismo

murciano-almeriense. Termomediterráneo semiárido. Tomillares basófilos y gipsícolas. Anthyllidetalia terniflorae (Rosmarinetea officinalis). Muy frecuente en la zona de estudio, aporta la mayor cobertura en las comunidades de tomillares-romerales.

\section{Chaenorrhinum grandiflorum (Cosson) Willk.}

ALMERÍA: Rambla de los Yesos, Alboloduy. 30S WF3198, $550 \mathrm{~m}$, en pastizales terofíticos efímeros. I. Bocio, J. Lorite et F. Valle. 15-3-95, GDAC 42354. Rambla de los Yesos, Alboloduy.
30S WF3198, $550 \mathrm{~m}$, en pastizales terofíticos efímeros. J. Lorite et R. Monterrubio. 23-3-97, GDAC 42355.

Endemismo murciano-almeriense. Termomediterráneo semiárido-árido. Pastizales terofíticos efímeros sobre terrenos yesíferos o materiales volcánicos. Stipion capensis (Brachypodietalia dystachae, Tuberarietea guttatae). Esta localidad es la única de la zona en la que existen afloramientos de yesos por los que se trata de una especie escasa.

\section{Coris hispanica Lange}

ALMERÍA: Prox. de Alsodux. 30S WF3595, $380 \mathrm{~m}$, tomillares subdesérticos. J. Lorite 27-4-96, GDAC 42349.

Endemismo murciano-almeriense: almeriense. Termomediterráneo semiárido. Tomillares subdesérticos sobre cárcavas. Anthyllido-Salsolion papillosae (Rosmarinetea officinalis).

\section{Euzomodendron bourgaeanum Cosson}

ALMERÍA: Prox. de Bentarique. 30S WF3394, $400 \mathrm{~m}$, tomillares subdesérticos. J. Lorite et R. Monterrubio. 21-2-96, GDAC 42346.

Endemismo murciano-almeriense: almeriense. Termomediterráneo semiárido. Tomillares basófilos y gipsícolas. Anabaso hispanicaeEuzomodendretum bourgeani (Anthyllido-Salsolion papillosae, Anthyllidetalia terniflorae, Rosmarinetea officinalis). Frecuente en la zona de estudio, aunque por sus requerimientos ecológicos restringida a algunos enclaves.

\section{Forskaolaea tenacissima $\mathrm{L}$.}

ALMERÍA: Rambla de los Yesos, Alboloduy. 30S WF3199, $500 \mathrm{~m}$, en tomillares nitrificados. I. Bocio, J. Lorite et F. Valle. 25-4-95, GDAC 42358.

Elemento de amplia distribución y diferencial en la Península Ibérica del murciano-almeriense: almeriense (en la Península, puesto que su distribución es bastante amplia extendiéndose por; Asia, Arabia, Asia Menor y Norte de África). Termomediterráneo semiárido. Comunidades nitrófilas y halonitrófilas. Onopordion nervosi (Carthametalia lanati, Onopordenea acanthi, Artemisietea vulgaris) y Salsolo vermiculataePeganion harmalae (Salsolo vermiculataePeganetalia harmalae, Pegano harmalae-Salsoletea vermiculatae).

Es bastante rara en la Península, en la zona 
solo hemos encontrado dos poblaciones en la Rambla de los Yesos.

Herniaria fontanesii Gay subsp. almeriana Brummitt et Heywood

ALMERÍA: Prox. de Alsodux. 30S WF3198, $370 \mathrm{~m}$, en tomillares subdesérticos. J. Lorite. 27-496, GDAC 42359. Rambla de los Yesos, Alboloduy. 30S WF3199, $600 \mathrm{~m}$, en tomillares subdesérticos, J. Lorite, 15-4-95, GDAC 42360.

Endemismo murciano-almeriense: almeriense. Termomediterráneo semiárido. Tomillares basófilos sobre pizarras margosas y conglomerados. Anthyllido-Salsolion papillosae (Anthyllidetalia terniflorae, Rosmarinetea).

\section{Launaea arborescens (Batt.) Murb}

ALMERÍA: Prox. de Bentarique. 30S WF3394, $400 \mathrm{~m}$, tomillares subdesérticos. J. Lorite et R. Monterrubio 21-4-96, GDAC 42347.

Es un elemento ibero-norteafricano de distribución restringida (N.W. de África y S.O de España, puntual en las Islas Canarias). En la Península su distribución se restringe a la provincia Murciano-Almeriense en el termomediterráneo semiárido, ocupando tomillares basófilos y penetra en formaciones de ramblas y halonitrófilas. Anthyllidetalia terniflorae (Rosmarinetea officinalis), Salsolo vermiculatae-Peganetalia harmalae (Pegano harmalae-Salsoletea vermiculatae) y Tamaricetalia africanae (NerioTamaricetea). Es frecuente aunque siempre aparece como ejemplares aislados.

\section{Limonium cossonianum Kuntze}

ALMERÍA: Rio Nacimiento, Alboloduy. 30S WG3300, $340 \mathrm{~m}$, en depresiones salinas. J. Lorite \& C. Salazar. 3-8-97, GDAC 42348.

SE ibérico y Baleares. Termomediterráneo semiárido. Tomillares sobre suelos yesosos-salinos. Lygeo sparti-Limonium furfuracei (Sarcocornietea fruticosae).

\section{Linaria oligantha Lange}

ALMERÍA: Loma del Juego de la Pelota, Instinción. 30S WF3095, $500 \mathrm{~m}$, en pastizales terofíticos efímeros. J. Lorite, J. Muñoz, T. Moral et R. Monterrubio. 21-3-96, GDAC 42350.

Endemismo murciano-almeriense. Termomediterráneo semiárido. Pastizales terofíticos efímeros. Stipion capensis (Brachypodietalia dystachae, Tuberarietea guttatae).

\section{Moricandia foetida Bourgeau ex Cosson}

ALMERÍA: Prox. de Alsodux. 30S WF3595, $380 \mathrm{~m}$, en tomillares subdesérticos, J. Lorite. 27-496, GDAC 42351. Prox. de Alsodux. 30S WF3595, $350 \mathrm{~m}$, en tomillares subdesérticos, J. Lorite. 27-496, GDAC 42352.

Endemismo murciano-almeriense: almeriense. Termomediterráneo semiárido-árido. Tomillares verticícolas y herbazales nitrófilos. Anabasio hispanicae-Euzomodendretum bourgeani (Anthyllido-Salsolion papillosae, Rosmarinetea officinalis), Carrichtero annuae-Amberboion lippi (Stellarietea mediae).

\section{Salsola papillosa Willk.}

ALMERÍA: Prox. de Alsodux. 30S WF3595, $390 \mathrm{~m}$, en tomillares subdesérticos. I. Bocio, J. Lorite et F. Valle. 25-4-95, GDAC 42357.

Endemismo murciano-almeriense: almeriense. Termomediterráneo semiárido-seco. Tomillares basófilos verticícolas, metalícolas y halonitrófilos. Anthyllido terniflorae-Salsolion papillosae (Anthyllidetalia terniflorae, Rosmarinetea officinalis) y Carthamo arborescentis-Salsolion oppositifoliae (Salsolo vermiculatae-Peganetalia harmalae, Pegano harmalae-Salsoletea vermiculatae).

\section{Sideritis pusilla (Lange) Pau subsp. pusilla}

ALMERÍA: Prox. de Alsodux. 30S WF3595, $380 \mathrm{~m}$, en tomillares subdesérticos. J. Lorite et R. Monterrubio. 27-4-96, GDAC 42356.

Endemismo murciano-almeriense: almeriense. Termomediterráneo semiárido. Tomillares basófilos. Helianthemo almeriensis-Sideritetum pusillae (Helianthemo almeriensis-Siderition pusillae, Anthyllidetalia terniflorae, Rosmarinetea officinalis). Aparece puntualmente en la zona.

\section{Teucrium almeriense Hubbard et Sandwith}

ALMERÍA: Rambla de los Yesos, Alboloduy. 30S WF3299, $550 \mathrm{~m}$, en tomillares subdesérticos. J. Lorite, 20-4-95, GDAC 42353.

Endemismo murciano-almeriense: almeriense. Termomediterráneo semiárido. Tomillares basófilos. Helianthemo almeriensis-Siderition pusillae (Anthyllidetalia terniflorae, Rosmarinetea offcinalis). 


\section{BIBLIOGRAFÍA}

CASTROVIEJO et al. (eds.) -1986/1997- Flora Iberica. Plantas Vasculares de la Península Ibérica e Islas Baleares. Vol. I-VIII. Jardín Botánico, CSIC. Madrid.

MOLERO MESA, J. y F. PÉREZ RAYA. -1987- La flora de Sierra Nevada. Avance sobre el catálogo florístico nevadense. Serv. Publ. Univ. Granada.

MOLERO MESA, J, F. PÉREZ-RAYA y F. VALLE -1992- Parque Natural de Sierra Nevada. Ed. Rueda. Madrid.

MOTA POVEDA, J.F. y F. VALLE TENDERO 1987- Estudio Botánico-Ecológico de las cuencas altas de los ríos Bayárcal, Paterna y Andarax. Servicio de publicaciones de la Diputación de Almería.
RIVAS-MARTÍNEZ, S., A. ASENSI, B. DÍEZ, J. MOLERO \& F. VALLE - 1997- Biogeographical synthesis of Andalusia (S. Spain). Journal of Biogeography 24: 915-928.

SAGREDO, R. -1987- Flora de Almería. Instituto de Estudios Almerienses. Almería.

TUTIN, T. et al. (eds.) -1964/1980- Flora europaea, Vols. 1-5. Cambridge University press. Cambridge.

Aceptado para su publicación en Abril de 1998

Dpto. Biología Vegetal, Facultad de Ciencias, Universidad de Granada. Aptdo. 18001 Granada. Email: fvalle@goliat.ugr.es

\title{
61. THYMUS VULGARIS L., NOVEDAD PARA LA FLORA DE ANDALUCÍA OCCIDENTAL
}

\author{
M. Ángeles MARTÍN MOSQUERO
}

Thymus vulgaris, a new specie in west Andalusia Flora.

Palabras clave. Lamiaceae, Thymus, Andalucía Occidental.

Key words. Lamiaceae, Thymus, west Andalusia.

Thymus vulgaris L. perteneciente a la Sect. Thymus Subsect. Thymus, es una especie que vive preferentemente sobre suelos ricos en bases, sobre todo en calizas y margas, también en terrenos yesosos cuando la influencia de éste no es muy acusada y raramente sobre suelos arenosos. Es frecuente encontrarlo en suelos pedregosos, grietas de rocas o sobre cantiles.

Son matas erectas o decumbentes, que se caracterizan, fundamentalmente, por sus tallos rojizos pubescentes con pelos cortos retrorsos, hojas lineares con margen revoluto, subpecioladas y sin cilios en la base, inflorescencias espiciformes acabezueladas y corolas de hasta $5 \mathrm{~mm}$ más o menos rosadas con anteras púrpuras.

Se distribuye por toda la región Mediterránea occidental europea, desde la Península Ibérica hasta Italia.

En la Península Ibérica está representada por dos subespecies; la subespecie vulgaris L. en la mitad $\mathrm{E}$, haciéndose escasa hacia el $\mathrm{S}$ 